\title{
Intercorrências e procedimentos neonatais no recém-nascido pré-termo tardio comparado ao recém-nascido a termo
}

\author{
Intercurrences and main neonatal procedures in the late preterm \\ newborn compared to newborn at term
}

\author{
Adriana Maria Tavares Gomes, ${ }^{1}$ Lilian Costa, ${ }^{1}$ Laís de Paiva Gabriel, ${ }^{2}$ Álvaro José Martins de Oliveira Veiga ${ }^{1}$ e \\ Adauto Dutra M. Barbosa ${ }^{3}$
}

${ }^{1}$ Faculdade de Medicina de Petrópolis Arthur Sa Earp Neto

¿Universidade Federal do Rio de Janeiro

${ }^{3}$ Universidade Federal Fluminense

\section{Palavras-chave:}

Nascimento prematuro; Prevalência; Morbidade.

\begin{abstract}
Resumo
Introdução: os recém-nascidos pré-termo tardios representam atualmente cerca de $70 \%$ do total de prematuros e, quando comparados aos recém-nascidos a termo, apresentam maiores riscos de complicações clínicas neonatais. Objetivo: comparar o perfil da morbidade neonatal dos recém-nascidos pré-termo tardios ao dos recém-nascidos a termo. Métodos: estudo transversal, descritivo, envolvendo todos os recém-nascidos com idade gestacional a partir de 34 semanas nascidos entre fevereiro e junho de 2016 na maternidade de um hospital universitário, divididos em dois grupos, de acordo com a idade gestacional - recém-nascidos pré-termo tardios (34 0/7 a 36 6/7 semanas) e recém-nascidos a termo (37 0/7 semanas ou mais) -, cujos dados foram coletados dos prontuários dos recém-nascidos e de suas mães. Foram calculadas a prevalência e a razão de chance de uma intercorrência ocorrer e de um procedimento ser realizado entre os recém-nascidos dos grupos. Resultados: a prevalência da prematuridade tardia foi de 8,8\%, de um total de 898 nascimentos. Quando comparado aos recém-nascidos a termo, os pré-termo tardios apresentaram maior chance de necessitar internação em UTI neonatal e assistência ventilatória $(R C=26,0)$, reanimação $(R C=3,0)$, de apresentar hipoglicemia ( $R C=9,8)$, icterícia neonatal com fototerapia $(R C=10,8)$, sepse neonatal $(R C=8,8)$, dificuldade de sucção ( $R C=13,8)$, necessidade de complementação alimentar $(R C=9,4)$ e coleta de sangue para exames complementares $(R C=22,5)$. Conclusões: os pré-termo tardios devem ser lembrados como recém-nascidos diferenciados que necessitam receber especial atenção durante seus cuidados, em virtude da maior frequência de morbidade a que estão sujeitos.
\end{abstract}

\section{Keywords:}

Premature birth; Prevalence; Morbidity.

\begin{abstract}
Introduction: late preterm newborns currently account for around $70 \%$ of all premature babies and, when compared to newborns at term, show an increased risk of neonatal complications. Objective: to compare the neonatal morbidity profile of late preterm newborn to newborn at term. Methods: a descriptive, cross-sectional study involving all newborns from the gestational age of 34 weeks, born between February and June 2016 in the maternity ward of an university hospital. They were divided into two groups, according to the gestational age, in late preterm newborns (34 0/7 to 36 6/7 weeks) and newborns at term (37 0/7 or more weeks), and the data was collected from the records of newborns and their mothers. The prevalence and the odds ratio of morbidity occurring in the groups was calculated. Results: the prevalence of late prematurity was $8.8 \%$, out of a total of 898 births. When compared to newborns at term, the late preterm newborns had more chances of requiring hospitalization in neonatal ICU and ventilatory assistance $(O R=26.0)$, resuscitation $(O R=3.0)$, and to present hypoglycemia $(O R=9.8)$, neonatal jaundice with phototherapy $(O R=10.8)$, neonatal sepsis $(O R=8.8)$, sucking difficulty $(O R=$ 13.8), food supplementation ( $O R=9.4)$ and need for blood collection for complementary tests $(O R=22.5)$. Conclusions: late preterm newborns should be remembered as newborns who need to receive special attention during their care, due to the greater frequency of morbidity to which they are subject.
\end{abstract}




\section{INTRODUÇÃO}

A taxa de prematuridade no Brasil está estimada atualmente em $11,5 \%$, sendo $74 \%$ destes pré-termo tardios (PMT-T). ${ }^{1}$

Os chamados 'pré-termo tardios' são aqueles recém-nascidos com idade gestacional (IG) entre 34 0/7 e 36 6/7 semanas. ${ }^{2}$ São comumente de tamanho e peso próximos aos daqueles nascidos a termo e, por isso, são muitas vezes atendidos pelos pediatras, incluindo neonatologistas, como se apresentassem desenvolvimento completo e baixo risco de morbidade. São frequentemente acompanhados em alojamento conjunto, sem rotinas diárias diferenciadas, e recebem altas precoces, ${ }^{3-5}$ com readmissão mais frequente..$^{6,7}$

Mesmo os prematuros tardios saudáveis apresentam risco significativamente maior de estar abaixo do peso no primeiro ano de vida, além de ter uma morbidade significativamente maior. ${ }^{8}$

O objetivo do presente trabalho foi descrever as principais intercorrências apresentadas e os principais procedimentos neonatais realizados no recém-nascido PMT-T, comparados aos do recém-nascido a termo (RNT).

\section{MÉTODO}

Estudo transversal, descritivo, envolvendo todos os nascimentos consecutivos de recém-nascidos com IG igual ou maior a 34 semanas no Hospital de Ensino Alcides Carneiro (HAC), da cidade de Petrópolis - RJ, entre fevereiro e junho de 2016.

Os recém-nascidos aceitos no estudo foram divididos em dois grupos, de acordo com a IG: PMT-T - aquele cuja IG fosse igual ou maior que 34 semanas e menor que 37 semanas completas; e RNT - aquele cuja IG fosse igual ou maior que 37 semanas. Foram excluídos todos os natimortos e os recém-nascidos portadores de malformações congênitas maiores.

Os dados foram obtidos dos prontuários maternos, de seus recém-nascidos e das declarações de nascidos vivos, por ocasião da alta, através do preenchimento de um formulário estruturado gerado no SurveyMonkey ${ }^{\circledR}$ software, contendo as variáveis de interesse, posteriormente exportado para o programa Microsoft Excel ${ }^{\circledR}$ v. 2015.

\section{ANÁLISE ESTATÍSTICA}

A análise estatística foi realizada no Programa Graph Pad Prism ${ }^{\circledast}$, com aplicação do teste exato de Fisher na análise estatística das variáveis categóricas e o teste $t$ de Student para as variáveis contínuas com distribuição normal, quando comparados PMT-T e RNT.

Calculou-se a prevalência da prematuridade tardia e a razão de chance de apresentar uma intercorrência ou de necessitar realizar um procedimento entre os recém-nascidos dos grupos.

O presente estudo foi aprovado pelo Comitê de Ética em Pesquisa da Faculdade de Medicina de Petrópolis sob o n. de Certificado de Apresentação para Apreciação Ética: 51737815.1.0000.5245.

\section{RESULTADOS}

Dos 898 recém-nascidos incluídos no estudo, 79 (8,8\%) constituíram o grupo PMT-T e 819 (91,2\%) constituíram o grupo RNT (Figura 1).

Não foram encontradas diferenças estatísticas entre os dois grupos com relação à média de idade materna: 26,75 \pm 7,10 anos no PMT-T, com idade mínima de 14 anos e máxima de 43 anos, versus $25,17 \pm 6,4$ anos no RNT, idade mínima de 13 anos e máxima de 44 anos (p $>0,05)$; ou com relação à via de parto: vaginal - 48,1\%

Figura 1 - Fluxograma da seleção da amostra em estudo

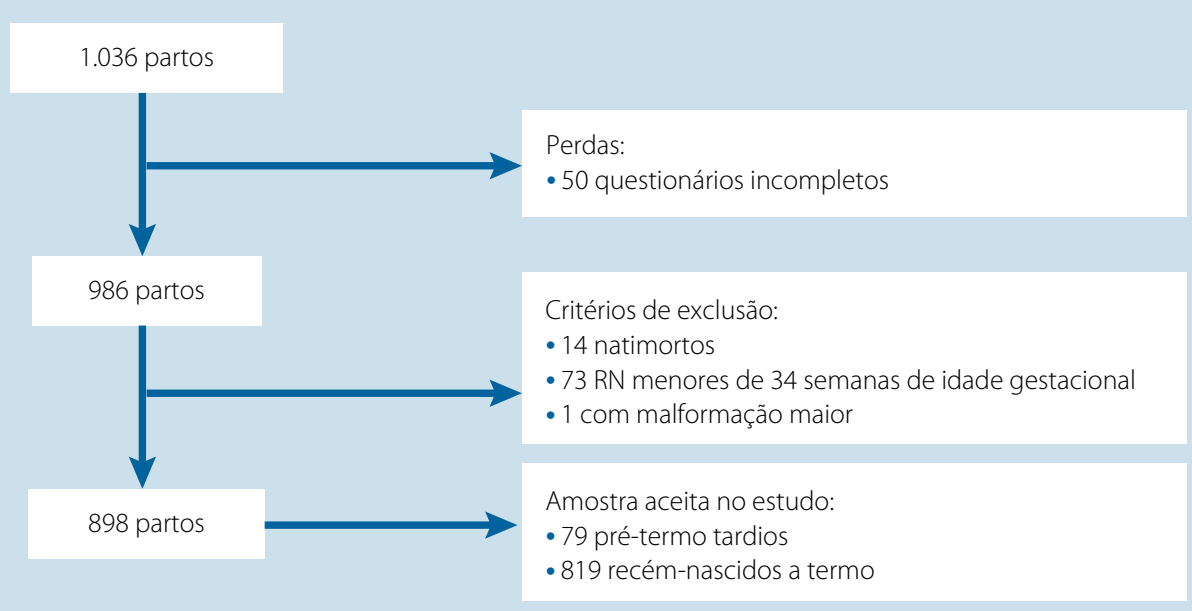

Fonte: Autoria própria (2018). 


\begin{tabular}{|c|c|c|c|}
\hline & $\begin{array}{l}\text { PMT-T } \\
n=79\end{array}$ & $\begin{array}{l}\text { RNT } \\
n=819\end{array}$ & $\mathbf{p}$ \\
\hline Peso $(\mathrm{g}) \pm \mathrm{DP}$ & $2.593 \pm 411$ & $3.295 \pm 476$ & $0,0001^{*}$ \\
\hline IG (semanas) $\pm \mathrm{DP}$ & $35,5 \pm 0,7$ & $39,2 \pm 1,4$ & $0,0001^{*}$ \\
\hline Apgar no 50 minuto \pm DP & $8.5 \pm 0,3$ & $9.1 \pm 0,7$ & $0,0001^{*}$ \\
\hline $\mathrm{TPH}$ (horas) $\pm \mathrm{DP}$ & $140,9 \pm 109,6$ & $76,4 \pm 61,9$ & $0,0032^{*}$ \\
\hline Sexo masculino & $27(34 \%)$ & 404 (49\%) & $0,013^{* *}$ \\
\hline
\end{tabular}

*Dados mostram significância estatística $-p<0,05$

*Não houve significância estatística - p>0,05.

PMT-T: pré-termo tardios; RNT: recém-nascidos a termo; DP: desvio-padrão; IG: idade gestacional; TPH: tempo de permanência hospitalar

versus cesariana $-56,8 \%(\mathrm{p}=0,5883)$.

Não se encontrou diferença estatística em relação ao grau de escolaridade materna entre os grupos, com média de 9,7 anos estudados pelas mães de PMT-T e 9,2 anos em mães do RNT ( $p>0,05)$.

Na tabela 1 estão relacionados os dados dos recém-nascidos quanto ao peso, à IG, ao índice de Apgar, ao sexo e ao tempo de permanência hospitalar (TPH).

A prevalência da prematuridade tardia foi de $8,8 \%$. Quando comparados aos RNT, os PMT-T apresentaram razão de chance (RC) de necessitar internação em unidade de terapia intensiva (UTI) neonatal e assistência ventilatória $(R C=26,0)$, reanimação $(R C=3,0)$, de apresentar hipoglicemia $(\mathrm{RC}=9,8)$, icterícia neonatal $(\mathrm{RC}=$ $10,8)$, sepse neonatal ( $R C=8,8)$, dificuldade de sucção $(\mathrm{RC}=13,8)$, necessitar complementação alimentar (RC $=9,4)$ e coleta de sangue para exames complementares $(\mathrm{RC}=22,5)$.

A tabela 2 apresenta dados das intercorrências neonatais e principais procedimentos realizados entre os dois grupos de estudo.

Morbidades tais como doença de membrana hialina (5,06\% PMT-T versus 0\% RNT), hipertensão pulmonar (1,27\% PMT-T versus $0,24 \% \mathrm{RNT})$, retinopatia da prematuridade (2,5\% PMT-T versus $0,12 \%$ RNT) e hemorragia intracraniana ( $1,27 \%$ PMT-T versus $0,12 \% \mathrm{RNT}$ ) não apresentaram diferença estatística significante $(p>0,05)$, enquanto foi observada diferença estatística para a pneumonia neonatal (3,8\% PMT-T versus $0,12 \%$ RNT; $p=0,002)$.

Quanto às causas de ocorrência dos partos prematuros foi observado uma maior prevalência de hipertensão gestacional, diabetes gestacional, ruptura prematura de membranas ovulares, vulvovaginites, corioamnionite, assim como um menor número de assistência adequada ao pré-natal (definido como $>6$ visitas) nas gestantes do grupo PMT-T quando comparadas às do grupo RNT $(p=0,05)$.

\section{DISCUSSÃO}

Nosso estudo corrobora com os achados da literatura, relacionados à morbidade e aos procedimentos envolvendo PMT-T e RNT. Verificamos que os PMT-T apresentam maior tempo de permanência hospitalar, incluindo o tempo de permanência na UTI neonatal, como tem sido descrito na literatura ${ }^{7}$ e isso se deve à presença de alguma morbidade muitas vezes associada a prematuros de menor IG. Observamos maior chance de hipoglicemia, ${ }^{9}$ especialmente no momento de transição à vida extrauterina, algumas vezes associada ao maior consumo de glicose gerado pelo estresse respiratório, ${ }^{10}$ outros casos devem-se à presença de sepse. ${ }^{11}$ Foi evidenciado também maior necessidade de suplementação de oxigênio ${ }^{12}$ através de pressão positiva contínua nas vias aéreas (CPAP) ou capacete (hood) e maior número de reanimações, ${ }^{13}$ quando comparado aos recém-nascidos a termo. Muitos prematuros evoluíram com dificuldade de sucção e/ou deglutição, por conseguinte, necessitando complementação alimentar. ${ }^{14}$

O PMT-T apresenta também com maior frequência quadros de icterícia, ${ }^{8}$ com indicação de uso de fototerapia. ${ }^{15}$ Embora os quadros de hipotermia pareçam ser mais frequentes entre os PMT-T, não encontramos diferença estatística entre os grupos quanto à ocorrência de hipotermia, o que nos leva a especular ser um achado possivelmente relacionado à assistência efetuada e não à IG.

De maneira similar aos resultados encontrados no presente estudo, Dani e colaboradores ${ }^{16}$ observaram elevado número de admissões de PMT-T na UTI neonatal, maior frequência de hipoglicemia e alterações na sucção e/ou deglutição. ${ }^{10}$ Dessa forma, os episódios de hipoglicemia, quase dez vezes mais frequentes nos nossos PMT-T, assim como o uso suplementar de oxigênio, ofertado através de capacete e/ou CPAP, explicam o elevado número de coleta de amostras sanguíneas, 
Tabela 2 - Intercorrências neonatais e principais procedimentos realizados entre os PMT-T e RNT

\begin{tabular}{|c|c|c|c|c|c|c|}
\hline & & $\begin{array}{l}\text { PMT-T } \\
n=79 \text { (\%) }\end{array}$ & $\begin{array}{l}\text { RNT } \\
n=819 \text { (\%) }\end{array}$ & $\mathbf{R C}$ & IC 95\% & $\mathbf{p}$ \\
\hline \multirow{2}{*}{$\begin{array}{l}\text { Internação em UTI (n. } \\
\text { pacientes) }\end{array}$} & $S$ & $22(27,8)$ & $12(1,5)$ & \multirow{2}{*}{26,0} & \multirow{2}{*}{12,2 a 55,1} & \multirow{2}{*}{$<0,0001$} \\
\hline & $N$ & $57(72,2)$ & $807(98,5)$ & & & \\
\hline \multirow{2}{*}{ Hipoglicemia } & $S$ & $30(38,0)$ & $48(5,9)$ & \multirow{2}{*}{9,8} & \multirow{2}{*}{5,7 a 16,9} & \multirow{2}{*}{$<0,0001$} \\
\hline & N & $49(62,0)$ & $771(94,1)$ & & & \\
\hline \multirow{2}{*}{$\begin{array}{l}\text { Fototerapia (icterícia } \\
\text { neonatal) }\end{array}$} & $S$ & $23(29,1)$ & $30(3,7)$ & \multirow{2}{*}{10,8} & \multirow{2}{*}{5,9 a 19,8} & \multirow{2}{*}{$<0,0001$} \\
\hline & $\mathrm{N}$ & $56(70,9)$ & $789(96,3)$ & & & \\
\hline \multirow{2}{*}{ Hipotermia } & $S$ & $4(5,1)$ & $13(1,6)$ & \multirow{2}{*}{3,3} & \multirow{2}{*}{1,1 a 10,4} & \multirow{2}{*}{0,0407} \\
\hline & $N$ & $75(94,9)$ & $806(98,4)$ & & & \\
\hline \multirow{2}{*}{$\begin{array}{l}\text { Sepse neonatal pre- } \\
\text { coce }\end{array}$} & $S$ & $13(16,5)$ & $18(2,2)$ & \multirow{2}{*}{8,8} & \multirow{2}{*}{4,1 a 18,7} & \multirow{2}{*}{$<0,0001$} \\
\hline & $\mathrm{N}$ & $66(83,5)$ & $801(97,8)$ & & & \\
\hline \multirow{2}{*}{ Dificuldade de sucção } & $S$ & $5063,3)$ & $91(11,1)$ & \multirow{2}{*}{13,8} & \multirow{2}{*}{8,3 а 22,9} & \multirow{2}{*}{$<0,0001$} \\
\hline & $N$ & $29(36,7)$ & $728(88,9)$ & & & \\
\hline \multirow{2}{*}{$\begin{array}{l}\text { Método alternativo } \\
\text { ou complementação } \\
\text { alimentar }\end{array}$} & $S$ & $66(83,2)$ & $287(35,0)$ & \multirow[b]{2}{*}{9,4} & \multirow[b]{2}{*}{5,1 a 17,6} & \multirow[b]{2}{*}{$<0,0001$} \\
\hline & $N$ & $13(16,8)$ & $532(65,0)$ & & & \\
\hline \multirow{2}{*}{ Reanimação } & $S$ & $15(19,0)$ & $59(7,2)$ & \multirow{2}{*}{3,0} & \multirow{2}{*}{1,7 a 5,6} & \multirow{2}{*}{0,0005} \\
\hline & $N$ & $64(81,0)$ & $760(92,8)$ & & & \\
\hline \multirow{2}{*}{ Oxyhood } & S & $41(51,8)$ & $105(12,8)$ & \multirow{2}{*}{7,4} & $45 a 110$ & < \\
\hline & N & $38(48,2)$ & $714(87,2)$ & & $4,3 \mathrm{~d} / 1,9$ & $<0,0001$ \\
\hline CPAP & S & $9(11,4)$ & $4(0,5)$ & 260 & 79 a 871 & (O) \\
\hline CPAP & $N$ & $70(88,6)$ & $815(99,5)$ & 20,0 & $1,9 \mathrm{~d} 8 /, 1$ & $<0,0001$ \\
\hline Colotac do & S & $74(93,4)$ & $325(39,7)$ & 275 & 00762 & \\
\hline Utelas ue sal igue & N & $5(6,6)$ & $494(60,3)$ & 22,3 & 9,0 a 56,2 & $<0,0001$ \\
\hline Óbito & & 0 & 0 & - & - & - \\
\hline
\end{tabular}

PMT-T: pré-termo tardios; RNT: recém-nascidos a termo; RC: razão de chance; IC: intervalo de confiança; CPAP: pressão positiva contínua nas vias aéreas.

que na nossa amostra foi realizada 20 vezes mais se comparada aos RNT e, consequentemente, aumentam o tempo de permanência hospitalar e elevam o número de casos de anemia entre os prematuros tardios. Esses achados são corroborados pela literatura. ${ }^{17,18}$

Teune et al. ${ }^{19}$ publicaram uma metanálise englobando mais de 29 milhões de partos, abordando mortalidade, morbidade e consequências a longo prazo na prematuridade tardia, comparada com o a termo. Kramer e colaboradores ${ }^{20}$ avaliaram todos os partos ocorridos nos Estados Unidos em 1995 e de 1992 a 1994 no Canadá. Ambos mostraram maiores riscos de mortalidade neonatal e infantil de forma relevante para o grupo de PMT-T, dados não corroborados pelo presente estudo, em que não ocorreram óbitos, refletindo possivelmente os cuidados dispensados a esses recém-nascidos no Serviço de Pediatria do HAC.

Outro aspecto importante a ser mencionado, embora não esteja no bojo deste estudo, é o elevado custo hospitalar gerado pela internação dos PMT-T, quando comparado àquele dos RNT, visto apresentar, como descrito, intercorrências clínicas importantes e necessidade de se empregar procedimentos complementares à rotina de atendimento normal ao recém-nascido.

\section{CONCLUSÕES}

Além de elevar o tempo de permanência hospitalar e da necessidade de assistência em UTI neonatal, a hipoglicemia, a icterícia neonatal e a ocorrência de taquipneia ocorrem mais frequentemente no PMT-T, quando comparado ao RNT, elevando a necessidade de complementação alimentar e o uso de CPAP ou de capacete, ocasionando necessariamente rastreamento infeccioso, com acentuada elevação no número de punções para coleta de amostras de sangue para os exames complementares pertinentes.

Os PMT-T devem ser lembrados como recém-nascidos diferenciados, que necessitam receber especial atenção durante seus cuidados, em virtude da maior frequência de morbidade a que estão sujeitos. 


\section{REFERÊNCIAS}

1 World Health Organization. Preterm birth. 19 February 2018 [accessed on Dec 10, 2018]. Available from: https://www.who. int/news-room/fact-sheets/detail/preterm-birth

2 Engle W, Tomashek K, Wallman C; Committee on Fetus and Newborn, American Academy of Pediatrics. "Late-preterm" infants: a population at risk [accessed on Dec 10, 2018]. Pediatrics. 2007;120(6):1390-401. Available from: http:// pediatrics.aappublications.org/content/120/6/1390.long

3 Leal MC, Esteves-Pereira AP, Nakamura-Pereira M, Torres JA, Theme-Filha M, Domingues RM et al. Prevalence and risk factors related to preterm birth in Brazil. Reprod Health [accessed on Dec 10, 2018]. 2016;13(Suppl 3):127. Available from: https://reproductive-health-journal.biomedcentral.com/ articles/10.1186/s12978-016-0230-0

4 McIntire D, Leveno K. Neonatal mortality and morbidity rates in late preterm births compared with births at term. Obstet Gynecol. 2008;111(1):35-41. DOI: https://doi.org/10.1097/01. AOG.0000297311.33046.73

5 Victora C. Intervenções para reduzir a mortalidade infantil pré-escolar e materna no Brasil. Rev Bras Epidemiol. 2001;4(1):3-69. DOI: http://dx.doi.org/10.1590/ S1415-790X2001000100002

6 Vohr B. Long-term outcomes of moderately preterm, late preterm, and early term infants. Clin Perinatol. 2013;40(4):739-51. DOI: https://doi.org/10.1016/j. clp.2013.07.006

7 Harron K, Gilbert R, Cromwell D, Oddie S, van der Meulen J. Newborn length of stay and risk of readmission. Paediatr Perinat Epidemiol. 2017;31(3):221-32. DOI: https://doi. org/10.1111/ppe.12359

8 Gupta P, Mital R, Kumar B, Yadav A, Jain M, Upadhyay A. Physical growth, morbidity profile and mortality among healthy late preterm neonates [accessed on Dec 10, 2018]. Indian Pediatr. 2017;54(8):629-34. Available from: https:// link.springer.com/article/10.1007/s13312-017-1123-1

9 Hosagasi NH, Aydin M, Zenciroglu A, Ustun N, Beken S. Incidence of hypoglycemia in newborns at risk and an audit of the 2011 American Academy of Pediatrics guideline for hypoglycemia. Pediatrics Neonatol. 2018;59(4):368-74. DOI: https://doi.org/10.1016/j.pedneo.2017.11.009

10 Kalyoncu O, Aygün C, Cetino lu E, Küçüködük S. Neonatal morbidity and mortality of late-preterm babies. J Matern Fetal Neonatal Med. 2010;23(7):607-12. DOI: https://doi. org/10.1080/14767050903229622

11 Goldstein ND, Eppes SC, Ingraham BC, Paul DA.
Characteristics of late-onset sepsis in the NICU: does occupancy impact risk of infection? J Perinatol. 2016;36(9):753-7. DOI: https://doi.org/10.1038/jp.2016.71

12 Natile M, Ventura ML, Colombo M, Bernasconi D, Locatelli A, Plevani C et al. Short-term respiratory outcomes in late preterm infants. Ital J Pediatr. 2014;40:52. DOI: https:// doi.org/10.1186/1824-7288-40-52

13 De Carolis MP, Pinna G, Cocca C, Rubortone SA, Romagnoli $\mathrm{C}$, Bersani I et al. The transition from intra to extra-uterine life in late preterm infant: a single-center study. Ital J Pediatr. 2016;42:87. DOI: https://doi.org/10.1186/s13052-016-0293-0

14 Giannì ML, Roggero $\mathrm{P}$, Piemontese $\mathrm{P}$, Liotto $\mathrm{N}$, Orsi A, Orsola Amato O. Is nutritional support needed in late preterm infants? BMC Pediatr. 2015;15:194. DOI: https://doi.org/10.1186/ s12887-015-0511-8

15 Sachdeva M, Murki S, Oleti TP, Kandraju H. Intermittent versus continuous phototherapy for the treatment of neonatal non-hemolytic moderate hyperbilirubinemia in infants more than 34 weeks of gestational age: a randomized controlled trial. Eur J Pediatr. 2015;174(2):177-81. DOI: https://doi. org/10.1007/s00431-014-2373-8

16 Dani C, Corsini I, Piergentili L, Bertini G, Pratesi S, Rubaltelli FF. Neonatal morbidity in late preterm and term infants in the nursery of a tertiary hospital. Acta Padiatr 2009;98(11):18413. DOI: https://doi.org/10.1111/j.1651-2227.2009.01425.x

17 McIntire D, Leveno K. Neonatal mortality and morbidity rates in late preterm births compared with births at term. Obstet Gynecol. 2008;111(1):35-41. DOI: https://doi.org/10.1097/01. AOG.0000297311.33046.73

18 Bettiol H, Barbieri MA, Silva AAM. Epidemiologia do nascimento pré-termo: tendências atuais [accessed on Dec 10, 2018]. Rev Bras Ginecol Obstet. 2010;32(2):57-60. Available from: http://www.scielo.br/pdf/rbgo/v32n2/v32n2a01.pdf

19 Teune MJ, Bakhuizen S, Gyamfi Bannerman C, Opmeer BC, van Kaam AH, van Wassenaer AG et al. A systematic review of severe morbidity in infants born late preterm. Am J Obstet Gynecol. 2011;205(4):374.el-9. DOI: https://doi. org/10.1016/j.ajog.2011.07.015

20 Kramer MS, Demissie K, Yang H, Platt RW, Sauvé R, Liston $\mathrm{R}$. The contribution of mild and moderate preterm birth to infant mortality. Fetal and Infant Health Study Group of the Canadian Perinatal Surveillance System [accessed on Dec 10, 2018]. JAMA. 2000;16:284(7):843-9. Available from: https:// jamanetwork.com/journals/jama/fullarticle/192994 